(7I) A. V. Markov, Pulkovo Bull. rg, no. I49, 64-81, 1952.

(72) N. N. Sytinskaya, Astr. Circ. U.S.S.R. no. I44, I I-I2, 1953.

(73) V. V. Sharonov, Bull. Len. Univ. no. 30, 3-7, 1952.

(74) N. S. Orlova, Ann. Len. Univ. 153, 166-94, 1952; Bull. Len. Univ. no. 32, 3-4, I95 I.

(75) A. P. Borissova and N. A. Budnikova, Bull. Len. Univ. 8.

(76) V. V. Sharonov, Astr. J. U.S.S.R. 3I, no. 5, 444-53, 1954.

(77) A. V. Markov, Pulkovo Bull. 19, part 2, no. 149, p. 65, 1952.

(78) I. Groeneveld and G. P. Kuiper, $A p . J$. 120, 200, 1954; $A p . J$. 120, 529, 1954.

(79) A. V. Shatzel, $A p . J$. 120, 547, 1954.

(80) I. I. Ahmad, $A p . J$. 120, 55I, 1954.

(8I) Ap.J. I20, 200, I954.

(82) B. J. Levin, Astr. Circ. U.S.S.R. no. I4I, 1953.

(83) G. P. Kuiper, Proc. Nat. Acad. Sci., Wash., 39, I I59, 1953; Ap. J. 120, 2 I9, 1954.

(84) O. J. Schmidt, C.R. Acad. Sci. U.R.S.S. 96, no. 3, I954.

(85) E. A. Lubimova and A. S. Starkova, Astr. J. U.S.S.R. 31, no. 5, I 954.

(86) G. P. Kuiper, Proc. Nat. Acad. Sci., Wash., 40, I Ior, I954.

(87) N. P. Barabashev, Investigation of the Physical Conditions on the Moon and the Planets (Kharkov Univ. 1952, 270 pp.).

(88) S. D. Gutshabash, Ann. Len. Univ. 1, I 53, 19-38, 1952.

(89) V. V. Sobolev, Progress in Astronomy, 6, 250-80, 1954.

(9o) V. V. Sharonov, C.R. Acad. Sci. U.S.S.R. 82, no. 3, 55I-3; Astr. J. U.S.S.R. 29, 728-37, 1952.

(91) V. V. Sharonov, Astr. Circ. U.S.S.R. no. 125, 8-9, 1952; Bull. Len. Univ. 8, no. 8, $5 \mathrm{I}-8 \mathrm{o}, 1953$.

(92) E. Rabe, Astr. J. 55, I I 2, 1950.

(93) H. C. Urey, Geochimica et Cosmochimica Acta, I, 23I, 195 I ; G. P. Kuiper, The Atmospheres, p. 339.

(94) Harold Jeffreys, The Earth (3rd ed. Cambridge, 1952).

(95) K. E. Bullen, Seismology (Methuen, I954).

(96) Sir Edward Bullard and B. Mason, The Earth as a Planet, chs. 3 and 6.

(97) G. P. Kuiper, Proc. Nat. Acad. Sci., Wash., 40, rog6, 1954.

(98) W. H. Ramsey, Monthly Notices, III, 427, 195 I.

(99) B. Miles and W. H. Ramsey, Monthly Notices, I12, 234, 1952.

(10o) W. C. De Marcus, Astr. J. 58, 36, r953.

(Ior) M. J. M. Bernal and H. S. W. Massey, Monthly Notices, Ir4, I72, I954.

(102) H. P. Berlage, Proc. Kon. Ned. Acad. Wet. 54, 344, 195 I.

(I03) H. C. Urey, The Planets, New Haven, 1952, p. I59f.

(I04) G. P. Kuiper, in The Atmospheres, ed. Kuiper, p. 340.

(105) W. C. De Marcus, Astr. J. 59, I16, I954.

(I06) S. V. Kozlovskaya, C.R. Acad. Sci. U.R.S.S. 92, no. 5, r953.

(107) E. A. Lubimova, Bull. Acad. Sci. U.R.S.S., Geophys. Ser., no. 2, 1952; no. 6, 1953.

(108) E. A. Lubimova, Publ. Geophys. Inst. 26, 1954.

(109) J. A. Jacobs, Trans. R. Soc. Canada, 47, 33, 1953; Ibid. 48, 33, 1954; Nature, 171, 835 , 1953; Trans. Am. Geophys. Union, 35, I6I, I954.

(1 10) G. P. Kuiper, J.R.A.S. Canada, 50, 57, 105, 158, 1956.

\title{
APPENDIX
}

\section{REPORT ON MARTIAN NOMENCLATURE}

At the Rome meetings Commission 16 formed a subcommittee, with M. Fournier as chairman, charged with formulating a simplified and adequate system for naming the Martian surface markings, both semi-permanent and transient (Trans. I.A.U. 8, 2I6-I7). M. Fournier prepared a ten-page memorandum which he sent on about I October r954 
to the members of the sub-committee. He proposed to reduce the list of over 550 names used in Antoniadi's monograph on Mars (included in his index on pp. 224-39) to those of features having a certain degree of permanence, numbering 400; and to replace the other names, of transient features, by symbols. The code suggested for the symbols was based on a division of the planetary surface into octants, four for each hemisphere, starting at $0^{\circ}$ longitude. The northern octants are called $I, 3,5,7$; the southern $2,4,6,8$. Four categories of features are distinguished: continental regions, islands, promontories, etc., by $A, B, C, \ldots$; seas, gulfs, narrows, etc., by $a, b, c, \ldots$; lakes, marshes, fountains, etc., by $\alpha, \beta, \gamma, \ldots$; and streaks (replacing 'canals') by two numbers, or, $02,03, \ldots$. In each case the order advances with increasing longitude within the octant. Thus, Aureum Cornu becomes $2 A$, Iani Sinus $2 d$, Jovis Lacus $3 \beta$, and Scythes $1-05$. Reference is made to Dr Fournier's full report for further details.

M. Fournier's proposals are the first important step toward bringing order in a subject that had become unwieldy. He knew the surface of Mars and the vast literature on it as no other, and was eminently qualified to separate the transient features from the more permanent. It seemed useful, however, to inquire whether the code adopted for the transient features was the most practical. MM. Van Biesbroeck and Kuiper suggested that M. Fournier consider an alternative system of coding in which co-ordinates to the nearest degree are used instead of octants (three digits for longitude, two for latitude), followed by a letter designating one of the four categories used by M. Fournier: $C$ for continents, $M$ (maria), $L$ (lakes, lacs), and $S$ (str.). Such a code would allow one to find the feature immediately on a map. M. Kuiper further thought that there might be a need for a Martian map with only the principal features named, and he suggested that M. Fournier select the twenty or so most important named features, which might suffice, e.g. in astrophysical investigations.

M. Fournier has prepared such a list, but he felt that most Martian observers would consider the number twenty quite inadequate. He therefore selected an additional thirty features, in the next class of prominence. Other members also expressed their wish to have the full list of 400 names essentially retained. Actually, there is no difference of opinion here. There is certainly a need for a map with all observed semi-permanent features and showing all names retained by M. Fournier; while there may also be a need for a simplified map, showing all features but names for only the principal ones. If the map would not bear the names themselves but only numbers, which would be identified in a legend, one could print three sizes of numbers (for the first 20 , the next 30 , and the remaining 350 , respectively), in which case a single map would suffice.

So far there is no consensus of opinion on the code to be adopted for the transient features. Several members of Commission 16 have expressed their views and from these it is regarded premature to make recommendations at this time. Instead it is proposed that the work of the sub-committee be continued; that $M$. Dollfus be co-opted and made its chairman; that the sub-committee be charged to work out proposals for a code of transient features; that it consider the lists of twenty and fifty prominent features selected by M. Fournier and recommend the use to be made of them; and construct a map showing the named features, possibly along the lines as indicated above.

The sub-committee and Commission I6 wish to record the debt of gratitude which planetary astronomy owes the late M. Fournier for his contribution toward the improvement of Martian nomenclature, a task which few astronomers would have been able to perform.

\author{
GERARD P. KuIPER \\ President of the Commission
}




\section{AdDendum to DRAFT RePort}

Before and during the meetings a few supplementary reports were received. Dr de Vaucouleurs called attention to the work of Woolley (r) on 'Monochromatic magnitudes of Mars, I954'; and to his recent monograph on this planet(2). Dr Kharadze reported that Dzhapiashvili investigated the light distribution in the earth's shadow and penumbra, and found that the intensity gradient in the penumbra is greater than that explained by the effect of the earth's atmosphere up to the height of $120 \mathrm{~km}$. Further, his results are in good accord with Danjon's conclusions about periodic light variations of the eclipsed Moon depending on the eleven-year sunspot cycle. Dzhapiashvili made some I500 photoelectric measures of lunar features on I48 nights; he concluded that the lunar maria are younger than the other parts of the crust and that new evidence was found for the volcanic origin of lunar formations.

The report by $A$. G. Wilson on the International Mars Committee and that by H. C. Urey on the Age of the Solar System are reproduced below in full.

International Mars Committee. In September of I953 at the instigation of Urey, Slipher, and others, an 'International Mars Committee' was formed to co-ordinate observations of Mars during the opposition of 1954 and I956. The purpose of the Committee was both to see that important Martian observational programmes received proper attention by astronomers equipped to make contributions and to co-ordinate co-operative programmes. Many of the results obtained in I954 were reported at the last meeting of the Committee and are being published in various journals $(3,4,5)$. However, the bulk of the work is still in the process of reduction.

The outstanding achievement of the Committee in I954 was the International Photographic Patrol which kept Mars under as continuous surveillance as possible from widely separated longitudes from May through September. The following observatories cooperated in the patrol:

Observatory, Pic du Midi, France Lamont-Hussey Observatory,

Orange Free State Helwan Observatory, Egypt
Kodaikanal Observatory, India Bosscha Observatory, Java Observatory at La Plata, Argentina Lowell Observatory, U.S.A.

In addition, extensive supplementary sets of photographs were taken at Lick, Mount Wilson and Union observatories. In all, 39,000 exposures were taken of Mars and the most complete record of surface and atmospheric changes ever made was obtained. The composite plate log has been published as part of the I954 International Mars Committee Report(6).

The Age of the Solar System. The maximum age of the meteorites has been determined with great precision by very brilliant work on the chemical and mass spectrographic analyses of the lead, potassium and argon, and the rubidium and strontium of iron and stony meteorites. The lead work was done by Patterson, Brown, Tilton and Inghram ( 7 ), and Patterson $(8,9)$. The radiogenic lead contents of the Canyon Diablo and Henbury meteorites are the lowest relative to $\mathrm{Pb}^{204}$ of all sources of lead investigated. It is assumed that this is primeval lead, since the iron meteorites contain very small amounts of uranium and thorium. Using these data in conjunction with the isotopic composition of lead from the stone meteorites, the ratio of $\mathrm{Pb}^{206}$ to $\mathrm{Pb}^{207}$, produced by $\mathrm{U}^{235}$ and $\mathrm{U}^{238}$ in these objects since the separation of the iron meteorites, can be calculated. The age is determined by this ratio and is found to be $4.5 \times 10^{9}$ years.

The $\mathrm{K}^{\mathbf{4 0}}-\mathrm{A}^{\mathbf{4 0}}$ ratio determines only a minimum age, since argon may have been lost by leakage and especially so if heating occurred. Gerling and Pavlova(ro) estimated the age of two meteorites as $3 \times 10^{9}$ years by this method. Krinov(II) secured smaller ages. Wasserburg and Hayden $\left(\mathbf{x}_{2}, \mathbf{r}_{3}, \mathbf{r}_{4}\right)$ have determined the ages of three chondrites and found ages between 4.66 and $4.82 \times 10^{9}$ years. The potassium analysis was made by the isotopic dilution method and the argon was analyzed mass-spectrometrically. Because of uncertainties in the decay constants these values may be as low as $4 \cdot 4 \times 10^{8}$ years. Urey (15, 16) 
has pointed out that these dates must be interpreted as the age of the solar system and has argued that Gerling and Pavlova's determination of potassium may be too high and hence a correction of their ages may give values comparable to the Wasserberg and Hayden results.

Ahrens and his co-workers( $\mathrm{x} 7$ ) have introduced the $\mathrm{Rb}^{87}-\mathrm{Sr}^{87}$ dating method. Schumacher(18) has found that the $\mathrm{Sr}^{87}$ isotopic abundance in the strontium of the achondrite, Pasamonte, is lower than that of any other known sample of strontium, and that the ratio of strontium to rubidium was very large. Assuming that this is the primeval abundance, the isotopic abundance of $\mathrm{Sr}^{87}$ in other meteorites and the rubidium content give an age of $5 \times 10^{9}$ years, with a considerable probable observational error and additional uncertainty because of the probable error of the decay constant.

Thus an age of the solar system of $4.5 \times 10^{9}$ years is indicated. This is a very important astronomical datum and now for the first time it can be regarded as reliable.

\section{REFERENCES}

(I) R. v. d. R. Woolley, Monthly Notices (in the Press).

(2) G. de Vaucouleurs, Physics of the Planet Mars (London, 1954).

(3) E. Pettit and R. S. Richardson, Publ. A.S.P. 67, 62, 1955.

(4) H. L. Johnson and A. J. Gardiner, Publ. A.S.P. 67, 74, 1955.

(5) W. M. Sinton, Sky and Telescope, 14, 360, 1955.

(6) E. C. Slipher and A. G. Wilson (eds.), Mars 1954, Report of the International Mars Committee (Flagstaff, Arizona, r955).

(7) C. C. Patterson, H. Brown, G. Tilton, and M. Inghram, Phys. Rev. 92, 1234, 1953.

(8) C. C. Patterson, Nuclear Processes in Geologic Settings (University of Chicago Press, I953), p. 36.

(9) C. C. Patterson, Geochimica et Cosmochimica Acta, 7, 151, 1955.

(Io) E. K. Gerling and T. G. Pavlova, Doklady Akad. Nauk, U.S.S.R. 77, 85, 1951.

(11) E. L. Krinov, Priroda Is. Akad. Nauk, U.S.S.R. Moscow, 40, no. 10, 83, 1951.

(12) G. J. Wasserburg and R. J. Hayden, Phys. Rev. 97, 86, 1955.

(13) G. J. Wasserburg and R. J. Hayden, Geochimica et Cosmochimica Acta, 7, 51, 1955.

(I4) G. J. Wasserburg and R. J. Hayden, unpublished work.

(I5) H. C. Urey, Nature, 175, 35I, I955.

(16) H. C. Urey, Proc. Nat. Acad. Sci. 4r, r27, 1955.

(17) L. H. Ahrens, Nuclear Geology, ed. H. Faul (New York: John Wiley and Sons, 1954), pp. 33I-4I.

(18) E. Schumacher, unpublished work.

Report of meetings, Commissions I6 and I6a. 31 August I955

President: Prof. G. P. Kuiper.

Secretaries: A. Dollfus and E. A. Whitaker.

Some corrections and additions to the Draft Report were discussed, after which the Report was approved by the Commission.

A number of new results were announced and discussed in the meeting. Dr Whipple reported that F. G. Smith of Cambridge had observed an occultation of part of the Gemini radio source by the Moon and that no evidence was found for the presence of a lunar ionosphere. This puts the upper limit of the lunar atmosphere at about $\mathbf{1 0}^{-\mathbf{1 2}}$ terrestrial atmospheres, $\mathrm{IO}^{3}$ lower than the limit established by Dollfus with the chronograph at the Pic-du-Midi. This result strengthens the conclusion that telescopic meteor trails attributed to the Moon are probably terrestrial in origin, although there appears to be some difficulty with the low velocities so estimated. This difficulty was stressed by 
Dr Luplau Janssen who reported extensive observations on this problem at Urania Observatory. The President recalled the I938 resolution (Trans. I.A.U. 6, p. 339) recommending the study of the equation of state of gases under high pressures and noted with pleasure the work now being done by J. Stewart at the University of Virginia, who is investigating the compressibility of material at $4^{\circ} \mathrm{K}$. up to pressures of $20,000 \mathrm{~atm}$. This work is of great potential importance in studies of planetary structure. Dr Burke of the Carnegie Institute of Washington reported on observations of the radio emission on Jupiter, discovered by Franklin and himself, and observed also at Sydney, Australia. The observations demonstrate the presence of one major (though variable) source and several minor sources. The Australian observations by Shain define the period of rotation for the major source: $P=9^{\mathrm{h}} 55^{\mathrm{m}} 13 \pm 5^{\mathrm{s}}$ (which is approximately that of System II of Jupiter), and further indicate the identification with a whitish cloud in the South Tropical belt, some $20^{\circ}$ jovicentric longitude in extent, for which Dr Alexander found the period of rotation to be $9^{\mathrm{h}} 55^{\mathrm{m}} \mathrm{I2}$ or $13^{\mathrm{s}}$. Dr Alexander pointed out that this period of rotation is rather unique on the planet so that the identification appears rather probable. It was confirmed by the observation of a satellite eclipsing the cloud, which caused a sudden disappearance of the signal; however, the signal did not reappear quite as soon as expected, which could be due to intrinsic fluctuations. The radio data defined an angle of emission in longitude of $I 35^{\circ}$, obtained from the signals as the source crossed the disk. The flux of the signal at $22 \mathrm{Mc}$. is $10^{-21}$ watts $/ \mathrm{m}^{2} \mathrm{sec}$. or a total emission of $10^{4}$ watts $/ \mathrm{sec}$., which is $10^{9}$ times that of a typical terrestrial lightning flash at the same frequency. The spectral distribution is very different from that of lightning, in the sense that it drops off much more rapidly toward the higher frequencies.

Dr A. G. Wilson briefly discussed the I 956 campaign of Mars observations and requested interested observers to communicate with him. Dr W. H. van den Bos circulated some excellent Mars photographs taken in 1954 by Finsen at Johannesburg. Dr H. K. Paetzold of Munich was determining the ozone distribution of our atmosphere from studies of lunar eclipses and urged astronomers to make spectroscopic observations of such eclipses and communicate with him on the results.

The problem of lunar nomenclature was discussed in connexion with recently published proposals for several additional names, though no such proposals had reached the Commission at this session. The following resolution was proposed by Dr Steavenson and unanimously adopted by the Commission:

The Commission recommends to the Union that at the present time-and particularly pending the completion of the proposed photographic map of the Moon-no official recognition shall be given to additional lunar nomenclature.

The atlas mentioned in the above resolution was briefly described by Dr Kuiper, who circulated a memorandum entitled: 'Considerations on a New Photographic Lunar Map'. Present plans call for a roo-inch lunar diameter, with forty sections covering the entire visible surface; each section is to be shown in at least three different illuminations. Dr Kuiper requested interested persons to communicate with him on any suggestions that might improve the usefulness of this undertaking. Dr Dollfus reported on the progress of the work of his sub-commission on Martian nomenclature. He reviewed the progress to date, mentioned that there was substantial agreement on dropping the I50 names of transient features and modifying certain others, as proposed by Fournier; but that the code to be adopted for the transient features was still under discussion. M. de Mottoni is drawing a series of charts based upon the Pic du Midi photographs to provide a mean 'definitive chart' and to determine which surface features are the most stable; while Dr Camichel is determining precise co-ordinates for both classes of features. 